\title{
COOPERATIVE SPECTRUM DECISION IN COGNITIVE VEHICULAR NETWORK BASED ON SUPPORT VECTOR MACHINE
}

\author{
Christopher Chembe ${ }^{1,2}$, Ismail Ahmedy ${ }^{1}$, Rafidah Md Noor ${ }^{1 *}$, Douglas Kunda', Michael Oche ${ }^{3}$, \\ Abubakar Bello Tambawal
}

\author{
${ }^{1}$ Department of Computer System and Technology, Faculty of Computer Science and Information \\ Technology, University of Malaya, Kuala Lumpur, Malaysia \\ ${ }^{2}$ School of Science, Engineering and Technology, Mulungushi University, Box 80415, Kabwe, Zambia \\ ${ }^{3}$ Department of Computing, Faculty of Science \& Technology. Kampala International University, Western \\ Campus, Ishaka, Uganda
}

Email: chembe@siswa.um.edu.my; ismailahmedy@um.edu.my; fidah@um.edu.my* (Corresponding author); dkunda@mu.ac.zm; ochesing@siswa.um.edu.my

DOI: https://doi.org/10.22452/mjcs.vol32no2.1

\begin{abstract}
Spectrum sensing is one of the core functionality of a cognitive radio system. Accurate identification of primary system signal can prevent interference and provide maximum protection to licensed users. However, many challenges associated with spectrum sensing still exist. Examples include shadowing, multipath fading of the primary user signal, hidden primary user problem and many more. To circumvent the challenges faced by sensing device, the cooperative decision from participating devices has been proposed in the literature. In this paper, we suggest a cooperative decision based on support vector machine (SVM) for spectrum sensing in vehicular communication environment to mitigate shadowing and multipath fading. Spectrum sensing can be formulated as a pattern recognition problem with sensing results grouped into two distinct classes corresponding to the presence or absence of a primary user signal. Therefore, we employ SVM at the fusion centre to classify the primary signal correctly. The roadside units along the highway are selected to act as the fusion centre where sensing decision is made. The performance of the proposed scheme is evaluated using receiver operation characteristic curves in MATLAB. We compare the probability of detection for the proposed cooperative scheme to hard fusion rule. The simulation result shows that SVM based cooperative decision performed better than hard fusion rule.
\end{abstract}

\section{Keywords: Spectrum Sensing, Cooperative Decision, Cognitive Radio, SVM, VANET}

\subsection{INTRODUCTION}

Vehicular Ad Hoc Network (VANET) has an important role in the Intelligent Transportation System (ITS) to safeguard the lives of people on the roads. Drivers can get online feedback about traffic conditions in advance to allow a smooth flow of traffic. On the other hand, passengers can experience a pleasant journey through entertainment provided by different service providers such as online peer-to-peer games, Internet Protocol Television (IPTV) [1], etc. VANET defines two types of communications to transmit messages for ITS applications [2]. First, vehicle to vehicle (V2V) communication and the second one is vehicle to infrastructure (V2I) communication. In V2V communication, links are established between two or more vehicles on the road in the ad hoc manner, while in V2I, vehicles establish communication links with stationary roadside units (RSU).

With an increase in the number of emerging applications and services developed for wireless technologies, demand for bandwidth has been growing steadily. For example, it is envisioned that by 2020 , there will be more than 50 billion devices connected to the Internet mostly through wireless communication [3]. This poses a major challenge on the already scarce radio frequency spectrum which is a finite resource. For instance, the Federal Communications Commission (FCC) in the USA has allocated almost all the channels in the communicable frequency bands to licensed users [4]. The unlicensed spectrum channels are reserved for Industrial, Scientific and Medical (ISM) usage and shared among different radio technologies such as Bluetooth, WiFi, Microwave ovens and emerging radio technologies. ITS applications have been allocated $75 \mathrm{MHz}$ at the $5.9 \mathrm{GHz}$ band for dedicated short range communication (DSRC) for V2V and V2I communications [5]. The $75 \mathrm{MHz}$ is divided into 7 channels with $10 \mathrm{MHz}$ each. $5 \mathrm{MHz}$ is reserved as guard band channel. Regardless, these channels can become congested, particularly during peak hours or accident scenarios, when the number of vehicles contending for the same channels increases [6]. In such situations, the delivery of delay sensitive safety and emergency messages becomes difficult. 
Different solutions have been proposed to overcome congestion of 7 DSRC channels during peak hours and traffic jams or accident scenarios. One such solution is to use protocols which guarantee the quality of service based on priority [7]. The safety messages are given high priority while service messages such as infotainment are given low priority. Other solution includes adapting modulation scheme at MAC layer to meet the minimum quality of service (QoS) requirement [38]. However, important service applications may suffer from this approach when there is high vehicle density [8]. This is because many vehicles will be contending for the 7 channels to transmit the high priority safety messages.

Cognitive radio (CR) technology has emerged as a promising solution to combat the scarcity of communication channels and provide bandwidth to emerging ITS applications through dynamic spectrum access (DSA). A CR is an intelligent software defined radio (SDR) that reconfigures its network parameters based on the network environment [9]. Preliminary work has shown that much of the licensed bands allocated to certain users are underutilized [10]. Unlicensed users, also called secondary users (SUs) can opportunistically access these underutilized radio frequency bands whenever the licensed users or primary users (PUs) are absent. In VANET, the SUs are vehicles on the road that can opportunistically use the licensed channels whenever there is congestion in the DSRC channels defined at the $5.9 \mathrm{GHz}$ band. However, there are many fundamental problems that need to be addressed before DSA can be integrated into a vehicular environment. One such problem is how to identify the underutilized radio spectrum in the licensed frequency bands. This challenge can be resolved through spectrum sensing. Spectrum sensing involves identification of free spectrum bands in frequencies of licensed users. Within the vehicular environment, there are challenges encountered in spectrum sensing including multipath fading and shadowing of the PU signal due to obstacles. Therefore, novel methods of detecting spectrum opportunities in Cognitive Vehicular Networks (CVN) must be developed. One way to identify the presence of the primary user (PU) signal in CVN is by embracing techniques that can classify the PU signal correctly. In this regard, machine learning algorithms are appropriate for deciding the presence or absence of the PU signal. Machine learning techniques have the potential to recognize patterns in data. Pattern recognition is used to classify a given set of data into different categories. Thus, spectrum sensing in CVN can be formulated as a pattern recognition problem. The PU signal in pattern recognition is classified as either present or absent. Consequently, classification algorithms based on machine learning can be applied. In recent years, machine learning algorithms have been proposed for spectrum sensing in cognitive wireless radio networks [11].

In this paper, we propose a cooperative decision for spectrum sensing in CVN based on support vector machine (SVM). SVM is a supervised machine learning technique that has been applied to data classification [12]. In the proposed scheme, individual vehicles perform sensing using energy detection technique. Once individual vehicles decide the spectrum occupancy locally, the results are sent to a central node (RSU) which constructs vectors of energy levels for classification. Cooperative decision at the RSU is proposed to mitigate multipath and shadowing experienced by individual vehicles. This is achieved through spatial and diversity gain obtained from participating vehicles cooperatively. Nevertheless, before online classification, the RSU passes through a training phase. This is done offline once a large number of training samples are collected from passing vehicles. The trained RSU classify subsequent test energy levels submitted from cooperating vehicles into distinct classes on which the decision of spectrum occupancy is made. In SVM, the training energy levels constructed at the RSU for training must be accompanied by a label indicating the class. Once the training energy vectors are obtained, different kernel functions are employed for learning purposes. The kernel functions are used to map input training energy vectors into high dimensional feature space to separate classes linearly. In this paper, we select three kernel functions; linear, polynomial and Gaussian radial basic function (RBF). We evaluate the performance of the proposed scheme using receiver operation characteristic (ROC) curves and compare the simulation results to the hard fusion rule at RSU.

The rest of the paper is organized as follows. In section 2, we review some related literature on sensing schemes proposed for CVN environment. In section 3, we present the analytical model for spectrum sensing and cooperative decision at RSU using SVM. The simulation results are presented in section 4 and section 5 concludes the paper.

\subsection{RELATED SENSING SCHEMES IN CVN}

In this section, we give an overview of spectrum sensing techniques proposed in the literature. In [13], a belief propagation scheme for the vehicular network in a highway scenario is proposed. Vehicles send belief messages about the presence of PU to its neighbors on which the cooperative decision is made. However, the model was validated using only 3 nodes. Authors in [14] examined the impact of mobility on spectrum sensing in CVN and proposed a cooperative spectrum management scheme for CVN. Their proposed sensing scheme is based on weighted majority correlation decision making. Vehicles on the highway are divided into cells to sense the licensed spectrum periodically. At regular intervals, vehicle broadcast messages to neighbors for correlation decision making. 
In [15], a distributed cooperative spectrum sensing which studies the receiver's uncertainty is proposed. The authors suggest a gradient based cooperative spectrum sensing in which each SU detects the channel energy level locally using energy detection techniques. The result of the observed signal is broadcasted to its immediate neighbors in the topology which has been authenticated using identifier based authentication protocol. Based on the energy received from its neighbors, each SU updates its energy status value to reach a consensus in an iterative manner, thereafter, the decision of the PU occupancy is made by individual SU. The schemes proposed are evaluated in V2V communication. The performance of the approaches described degrades with a low number of vehicles participating in the cooperative decision. In addition, they demand more bandwidth to satisfy the iterative communication of sensing results before a consensus of sensing results is reached [13]. These schemes differ from the proposed approach in this paper where our approach is based on learning as both previously learned results and current sensing results are used in deciding the PU occupancy state. Therefore, it performs better even in low vehicle density. In addition, as more vehicles participate in sensing, the accuracy of predicting the right category of PU signal state also increases. The learning can be done offline to increase the efficiency for RSU.

Authors in [16] recommended an infrastructure based spectrum sensing technique for the vehicular environment to identify ISM bands along the highway. A study by [17] explored challenges in using wideband sensing to detect PU activity and impact of vehicle mobility on time of spectrum sensing against the complexity of algorithms. Authors in [18] identified synchronization of sensing outputs as a challenge in infrastructure based cooperative sensing. This is attributed to the high mobility of vehicles making it difficult to synchronize sensing results. This is because samples of the same channel can be collected at different time and location by the same vehicle. To overcome the synchronization challenge, they recommended asynchronous cooperative spectrum sensing based on centralized and distributed implementation. The proposed scheme exploits the spatial and temporal diversities for spectrum sensing and access in a cognitive vehicular environment. In [35], a spectrum sensing which predicts availability channel is proposed. The scheme uses prior knowledge of channel availability probability and Bayesian inference to predict free channels in the future. Other cooperating sensing schemes proposed based on hard fusion rule includes [33] and [34]. In hard fusion, only one bit ( 1 or 0$)$ is sent to the fusion center for determining the PU occupant state. Nevertheless, these schemes also suffer some setbacks. In low vehicle density, the performance is not optimal because they base their decision on current sensing results. This is opposed to the machine learning approach in which previously learned sensing results are used to refine the decision. With more vehicles participating in the cooperative decision, the machine learning approach increases its decision accuracy.

Another approach to acquiring radio frequency from licensed channels is by constructing a spectrum availability database [36]. The spectrum database approach was proposed to provide maximum protection to licensed users. In this approach, vehicles on the road equipped with Internet or geo-location capabilities are permitted to query the spectrum databases. In most cases, the licensed channels that can be populated in the database include TV channels and wireless microphone bands [36]. To implement the database approach, three modes of devices were proposed [37]. The first called Mode II includes vehicles with Internet capabilities to query the database. The second mode includes vehicles that depend on Mode II vehicles to get free spectrum. The third mode includes sense-only vehicles. Therefore, even in the presence of database approach spectrum sensing is promoted. Furthermore, database query overhead is introduced if there are more vehicles accessing the database. In addition, the cost and time of creating and maintaining the spectrum database are disadvantageous. Hence, the method proposed in this paper can help alleviate spectrum scarcity through accurate spectrum sensing. Our approach localizes spectrum results and no querying the database through the Internet is needed. A comprehensive spectrum sensing approaches and open issues in CVN has been reported in [39].

In recent years, cooperative spectrum decision based on machine learning has been proposed for cognitive radio networks (CRN). For instance, a study by [19] proposed machine learning techniques in cognitive radio networks. They investigated the performance of both supervised and unsupervised learning. Simulation results showed that SVM performed better than the K-nearest-neighbor method for supervised learning. Conversely, K-mean clustering performed better than Gaussian mixture model method for unsupervised learning. Other implementations based on SVM are presented in [20-22]. In [21], SVM is combined with genetic algorithms and self-organizing maps to achieve better sensing performance. In another study by [22], they proposed an eigenvalue based spectrum sensing with SVM in a multi-antenna cognitive radio. A reinforcement learning based cooperative sensing is proposed in [23] to address the cooperation overhead problem. Cooperative decision techniques based on machine learning have shown some improvements in obtaining accurate sensing results. Techniques based on machine learning have not been applied to spectrum sensing in CVN. Hence, we aim to exploit support vector machine, which is a supervised machine learning technique to classify sensing results in order to improve detection performance in CVN. Improved detection accuracy will protect the PU system from interference that may be caused by unlicensed users. Machine 
learning techniques are applied to cooperative spectrum sensing decision in CVN environment while taking VANET characteristics (e.g. vehicle speed) into consideration.

\subsection{SYSTEM MODEL}

This section describes the network model and spectrum sensing for both single vehicle and cooperating decision based on SVM. Furthermore, we outline the assumptions made in the formulation of the proposed model.

\subsection{Network model}

We assume that the vehicle is equipped with two antennas, one antenna to work with IEEE802.11p/WAVE to operate on $5.9 \mathrm{GHz}$ DSRC frequency bands. Another antenna configured with a CR to communicate over other licensed frequency bands when the $5.9 \mathrm{GHz}$ channels are congested. Primary users (PUs) are licensed TV bands users while vehicles are secondary users (SUs) that opportunistically access the TV bands. We consider a highway scenario divided into distinct segments, each segment is covered by a roadside unit (RSU). Fig. 1 illustrates this notion.

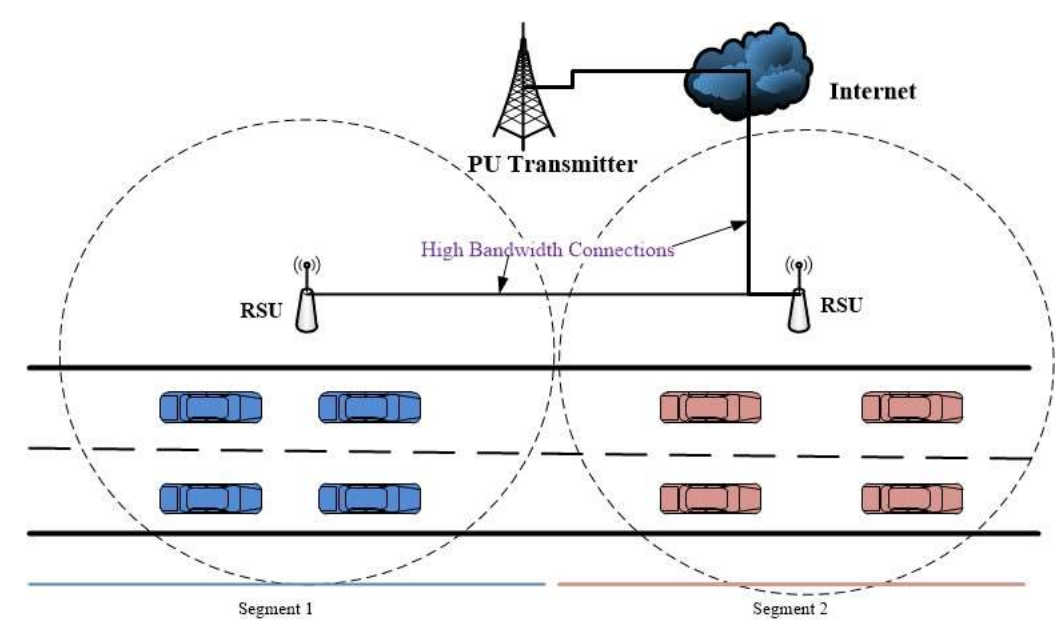

Fig. 1: Cognitive vehicle network

When a vehicle enters a congested road segment, it performs sensing for available free channels from licensed frequency bands that it can use for communication. The outcomes of the individual sensing will be sent to RSU on the common control channel of DRSC. We assume that the channels between vehicles and RSU are independent and identically distributed (i.i.d) and vehicle follows the freeway mobility model [24]. The sensing channel between the PU and SUs follows a correlated Rayleigh channel.

In congested road segments, vehicles move at a relatively slow velocity varying from 0 to $10 \mathrm{~km} / \mathrm{h}$ in highly dense traffic jams and up to $40 \mathrm{~km} / \mathrm{h}$ in medium dense road segments [25]. The transmission range for each RSU is assumed to be $300 \mathrm{~m}$ [26]. Hence, a vehicle in a medium to dense traffic is expected to be within RSU coverage between 27 seconds when moving at $40 \mathrm{~km} / \mathrm{h}$ to 108 seconds when moving at $10 \mathrm{~km} / \mathrm{h}$ (i.e. time = distance/ speed(velocity)). This can be considered to be enough time for vehicles to identify spectrum and communicate on the identified channels.

\subsection{Per-Vehicle sensing with energy detection}

Energy detector is the most commonly used spectrum sensing technique, due to its low computational complexity. In addition, prior knowledge of the PU signal is not needed. The spectrum occupancy of the PU is determined by measuring the signal power on the target frequency bands. Subsequently, the measured results are compared to a predefined threshold for that frequency band. The energy detector is very fast in detecting the presence or absence of the PU signal because it relies on the received energy signal. The fast detection property of energy detector makes it well suited for VANET environment where vehicles need to detect spectrum availability in a prompt and timely manner.

Each vehicle in the model can only sense one channel over the sensing time interval $\mathrm{T}$ in the segment. During the sensing period, $M$ samples are collected. For each segment, there are $N$ vehicles participating in the spectrum 
sensing decision. The decision of the PU signal on the individual vehicle is based on the Neyman-Pearson and Bayes binary hypothesis test defined as [27]:

$$
\mathrm{z}_{\mathrm{i}}(\mathrm{k})= \begin{cases}\mathrm{n}(\mathrm{k}) & \mathrm{H}_{0} \\ h r_{i}(\mathrm{k})+\mathrm{n}(\mathrm{k}) & \mathrm{H}_{1}\end{cases}
$$

for $i=1,2,3, \ldots, N$ and $k=1,2,3, \ldots, M, z_{i}(k)$ is the signal received by the ith vehicle. The signal transmitted by the PU, is given by $r_{i}(k)$ and $h$ represent the antenna gain between SU and PU. The $n_{i}(k)$ is the additive white Gaussian noise, which is i.i.d with mean zero and variance $\sigma_{n}^{2}$ (i.e., $\mathrm{n}_{i}(k) \sim \mathrm{N}\left(0, \sigma_{n}^{2}\right)$ ). The presence and absence of the PU signal is given by $H_{1}$ and $H_{0}$ respectively. The test energy statistic $d_{i}$ for each vehicle can be formulated as:

$$
d_{i}=\sum_{k=1}^{M}\left|z_{i}(k)\right|^{2}
$$

For sufficiently large sample size of $M \geq 250$ observed within a short sensing duration, $d_{i}$ can be approximated using central limit theorem (CLT) as a Gaussian distribution [28]. Therefore, the power density function (PDF) of $d_{i}$ at vehicle $i$ is approximated as:

$$
d_{i} \sim \begin{cases}\mathrm{N}\left(M \sigma_{n}^{2}, M \sigma_{n}^{4}\right) & \text { for } H_{0} \\ \mathrm{~N}\left[\left(M+\mu_{i}\right) \sigma_{n}^{2},\left(M+\mu_{i}\right) \sigma_{n}^{4}\right] & \text { for } H_{1}\end{cases}
$$

where

$$
\mu_{i}=\sum_{k=0}^{M-1}|h|^{2} \frac{E_{S}}{M \sigma_{n}^{2}}
$$

In Eq. 4 , the value of $E_{s}$ is equal to $\sum_{k=1}^{M}\left|r_{i}(k)\right|^{2}$ which is the transmitted energy signal of the PU for $M$ samples of each detection interval. All vehicles report the estimated energy signal to the fusion center where the spectrum occupancy is determined. The fusion center constructs the energy levels estimated at each vehicle into an energy vector as,

$$
X=\left(d_{1}, d_{2}, d_{3}, \ldots, d_{i}, \ldots, d_{N}\right)^{T}
$$

where $T$ is the transpose of the energy vector.

\subsection{Primary User detection model}

The core function of spectrum sensing is to determine whether primary user signal is present or absent in the given road segment in order to avoid interference to licensed users. Therefore, we formulate the PU occupancy as a classification problem. We assume the PU occupancy follows an ON/OFF activity model. For each ON period $\left(H_{1}\right)$, the PU is assumed to be active and no SU can transmit on that channel. The OFF period $\left(H_{0}\right)$ represents an instance when the PU is idle in which case SU can communicate on the channel. Hence, we can group the PU occupancy state into two availability classes (ON and OFF) for a given road segment $S_{j}$ as:

$$
Y=\left\{\begin{aligned}
-1 & \text { if no } P U \text { exist in } S_{j} \\
1 & \text { if } P U \text { exist in } S_{j}
\end{aligned}\right.
$$

The OFF period corresponds to channel availability represented by $Y=-1$ and ON period corresponds to channel unavailable given by $Y=1$. In our model, $Y$ is a label that is used in training phase for SVM. For each test statistic $X_{i} \in\left\{d_{1}, \ldots d_{i}, \ldots d_{N}\right\}$ of vehicles obtained from Eq. 2, there is an accompanying label $Y_{i} \in\{1,-1\}$ which determines the occupancy state of the PU signal. Therefore, $Y$ is the label sent to RSU determined by each vehicle based on individual sensing results (either $H_{1}$ or $H_{0}$ ). If the vehicle determines the presence of PU signal (i.e. $H_{1}$ ) locally based on energy detector (Section 3.2) then the label $Y$ will be 1 . On the other hand, if the vehicle determines the absence of the PU signal (i.e. $H_{0}$ ) locally then the label $Y$ will be -1 . Hence, the corresponding vector at RSU will be $Y=\left(Y_{1}, Y_{2}, Y_{3}, \ldots, Y_{i}, \ldots, Y_{N}\right)^{T}$ for $Y_{i} \in\{1,-1\} . N$ represents the total number of vehicles participating in sensing.

\subsection{Cooperative decision at RSU using SVM}

The proposed cooperative decision at RSU serves to correctly classify PU channel availability given the test energy vector. Prior to online classification, the RSU has to be trained to classify the subsequent test energy vectors correctly. 
Let $D=\left\{\left(X_{1}, Y_{1}\right),\left(X_{2}, Y_{2}\right), \cdots,\left(X_{N}, Y_{N}\right)\right\}$ be the training data, such that $X_{i} \in\left\{d_{1}, \ldots d_{i}, \ldots d_{N}\right\}$ and $Y_{i} \in\left\{Y_{1}, \ldots Y_{i}, \ldots Y_{N}\right\}$ for $i=1,2,3, \ldots N$, where $N$ corresponds to the total number of training samples obtained from individual vehicles participating in the cooperative decision. $X_{i}$ is a training energy value for the individual vehicle and $Y_{i}$ is the corresponding availability label determined by the vehicle at the local level after sensing. The label $Y=1$ represents the presence of the PU while $Y=-1$ denotes the absence of any PU in $S_{j}$. The goal of SVM is to find the optimal separating hyperplane between the positive and negative classes given by $\frac{2}{\|w\|}$, as shown in Fig. 2.

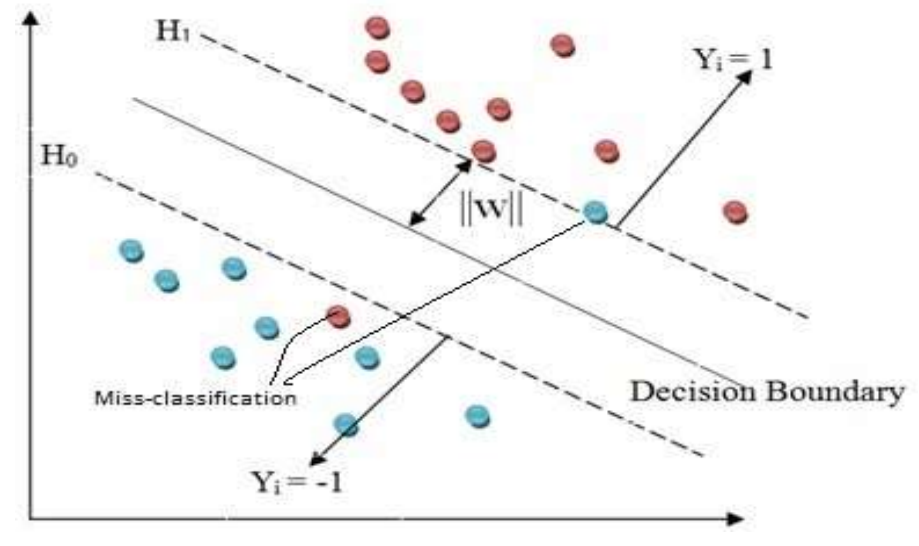

Fig. 2: SVM concept example

However, in low SNR, the training samples are not separated linearly [22]. Thus, we introduce a non-linear kernel function $\emptyset(X)$ that will map the training data into higher dimensional function space in order to linearly separate the classes. Therefore, the classifier should satisfy the following conditions:

$$
\begin{array}{ll}
\left\langle w . \emptyset\left(X_{i}\right)\right\rangle+\beta \geq 1 & \text { for } Y_{i}=1 \\
\left\langle w . \emptyset\left(X_{i}\right)\right\rangle+\beta \leq-1 & \text { for } Y_{i}=-1
\end{array}
$$

where $w$ is the weighing vector, $\beta$ is the bias that moves the hyperplane away from the origin and the quantity $\langle$.$\rangle is$ the inner product. Eq. 7 can be rephrased as:

$$
Y_{i}\left(\left\langle w . \emptyset\left(X_{i}\right)\right\rangle+\beta\right) \geq 1 \quad \forall i
$$

It is difficult to achieve ideal linearly separable hyperplane that satisfies Eq. 8 for each training data set. Therefore, to avoid overfitting the data, a slack variable $\rho_{i}$ is introduced for possible classification errors. Hence, Eq. 8 becomes

$$
Y_{i}\left(\left\langle w . \emptyset\left(X_{i}\right)\right\rangle+\beta\right) \geq 1-\rho_{i} \quad \forall i, \rho_{i} \geq 0
$$

The training samples for which $\rho_{i}=0$ are considered to be classified correctly and those that lie inside $0 \leq \rho_{i} \leq 1$ are considered to be within marginal classification errors and are on the correct side of the decision boundary. On the other hand, if $\rho_{i} \geq 1$, it is considered a classification error, this is illustrated in Fig. 2 . Therefore, the goal is to minimize the sum error while maximizing the margin of the classifier. This can be achieved as follows

$$
\begin{gathered}
\min \frac{1}{2}\|w\|^{2}+\theta \sum_{i=1}^{L} \rho_{i} \text { Subject to } Y_{i}\left(\left\langle w . \emptyset\left(X_{i}\right)\right\rangle+\beta\right) \geq 1-\rho_{i}, \\
\rho_{i} \geq 0, \mathrm{i}=1,2, \ldots, N
\end{gathered}
$$

where $\|w\|^{2}$ is the inner product given by $w^{T} . w$ while $\theta$ is a soft margin constant [29]. The resulting optimization problem is called a convex optimization problem and can be solved using Lagrangian function $\mathcal{L}$ as follows:

$$
\mathcal{L}(w, \beta, \rho, \alpha, \delta)=\left\{\begin{array}{c}
\frac{1}{2}\|w\|^{2}+\theta \sum_{i=1}^{N} \rho_{i}- \\
\sum_{i=1}^{N} \alpha_{i}\left[Y_{i}\left(\left\langle w . \emptyset\left(X_{i}\right)\right\rangle+\beta\right)-1+\rho_{i}\right]- \\
\sum_{i=1}^{N} \delta_{i} \rho_{i}
\end{array}\right\}
$$

where $\alpha_{i}$ and $\delta_{i}$ are Lagrangian multipliers. The training samples where $\alpha_{i}>0$ are called support vectors, and lies on one of the two hyperplanes. When Karush-Kuhn-Tucker (KKT) condition is applied to Eq. 11, the following can be deduced: 


$$
\begin{aligned}
& w=\sum_{i=1}^{N} \alpha_{i} Y_{i} \emptyset\left(X_{i}\right) \\
& \sum_{i=1}^{N} \alpha_{i} Y_{i}=0 \\
& \alpha_{i}+\delta_{i}=\theta
\end{aligned}
$$

Since $\alpha_{i} \geq 0$, we have $0 \leq \alpha_{i} \leq \theta$ with $\theta$ setting the upper bound. In addition, since the values of $\alpha_{i}$ are support vectors, the dual form of the problem defined in (11) in terms of support vectors can be formulated as follow [29]:

$$
\mathcal{L}(w, \beta, \rho, \alpha, \delta)=\left[\sum_{i=1}^{N} \alpha_{i}-\sum_{i=1}^{N} \cdot \sum_{j=1}^{N} Y_{i} Y_{j} \alpha_{i} \alpha_{j}\left\langle\emptyset\left(X_{i}\right), \emptyset\left(X_{j}\right)\right\rangle\right]
$$

Subject to $\sum_{i=1}^{N} Y_{i} \alpha_{i}=0,0 \leq \alpha_{i} \leq \theta$ for $\mathrm{i}=1,2 \ldots \mathrm{N}$

By solving the convex optimization problem in the Eq. 15 and applying the quadratic programming algorithm, the nonlinear decision function is obtained as follows:

$$
Y(X)=\operatorname{sgn}\left(\sum_{i=1}^{N} \alpha_{i} Y_{i} k\left(X, X_{i}\right)+\beta\right)
$$

where $\operatorname{sgn}$ is the sign function and $k\left(X, X_{i}\right)=\left\langle\emptyset\left(X_{i}\right), \emptyset\left(X_{j}\right)\right\rangle$ is the kernel function. Different types of kernel function can be used, including linear, polynomial and Gaussian radial basis function [29]. The linear kernel function is given by: $k\left(X, X_{i}\right)=X_{i}^{T} X_{j}$, polynomial kernel: $k\left(X, X_{i}\right)=\left(X_{i}^{T} X_{j}+1\right)^{d}, d>1$ and the radial basic function (RBF) kernel: $k\left(X, X_{i}\right)=\exp \left(-\frac{\left\|X_{i}-X_{j}\right\|^{2}}{2 \sigma^{2}}\right)[29]$.

\subsection{PERFORMANCE AND SIMULATION ANALYSIS}

We evaluate the performance of the energy detector and kernel of SVM in terms of probability of detection over the Receiver Operating Characteristics (ROC) curves using MATLAB simulator. The simulation uses models defined in Section 3. The PU activity follows the ON and OFF model. The ON period corresponds to when the PU is transmitting on the licensed channels and the OFF period denotes the inactive PU. The probability of detecting the PU signal is given by $P_{d}$ while the probability of false alarm detection is given by $P_{f} . P_{d}$ refers to an instance when the PU signal is correctly identified given the test energy level $d_{i}$ (Eq. 2) and some threshold $\lambda$. Hence $P_{d}=$ $\operatorname{Pr}\left(d_{i} \geq \lambda \mid H_{1}\right)$ and $P_{f}=\operatorname{Pr}\left(d_{i}<\lambda \mid H_{0}\right)$ [30]. Based on the energy statistics $d_{i}$, the $P_{d}$ and $P_{f}$ of a vehicle at the local level can be assessed as follows [31]:

$$
P_{f}=\frac{\Gamma\left(M / 2, \lambda / 2 \sigma^{2}\right)}{\Gamma(M / 2)}
$$

and

$$
P_{d}=Q_{\frac{M}{2}}\left(\sqrt{2 \mu_{i} / \sigma^{2}}, \sqrt{\lambda / \sigma^{2}}\right)
$$

where $\Gamma(.,$.$) defines the incomplete gamma function and Q_{M / 2}(.,$.$) is the standard Gaussian Marcum Q-function$ given by:

$$
Q(a)=\frac{1}{\sqrt{2 \pi}} \int_{a}^{\infty} \exp \left(\frac{-t}{2}\right) d t
$$

We assumed that the channel between the SU and the PU follows the Rayleigh distribution in the VANET environment. Therefore, the signal to noise ratio (SNR) $\mu$ complies with the exponential PDF of the Rayleigh channel fading [32]:

$$
f\left(\mu_{i}\right)=\frac{1}{\bar{\mu}} e^{-\frac{\mu_{i}}{\bar{\mu}}}, \mu \geq 0
$$

where $\bar{\mu}$ is the average SNR of the primary signal detected by the vehicle during the sensing interval. Making use of the integration of the Q-function, the average probability of detection defined in Eq. 18, can be averaged over Eq. 20 to give [31]: 


$$
\bar{P}_{d}=\exp \left(-\frac{\lambda}{2 \sigma^{2}}\right) \sum_{i=0}^{\frac{M}{2}-2} \frac{\left(\frac{\lambda}{2 \sigma^{2}}\right)^{i}}{i !}+\left(\frac{2 \sigma^{2}+\bar{\mu}}{\bar{\mu}}\right)^{\frac{M}{2}-1} *\left[\exp \left(-\frac{\lambda}{2 \sigma^{2}+\bar{\mu}}\right)-\exp \left(-\frac{\lambda}{2 \sigma^{2}}\right) \sum_{i=0}^{\frac{M}{2}-2}\left(\frac{\frac{\lambda \bar{\mu}}{4 \sigma^{4}+2 \sigma^{2} \bar{\mu}}}{i !}\right)\right]
$$

Note that the $P_{f}$ in Eq. 17 is not affected by Rayleigh fading because it is independent of the SNR transmitted by the PU, thus it remains unchanged.

The Q-function and Rayleigh function are already defined in MATLAB simulator. The rest of the simulation parameters are given in the following table:

Table 1: Simulation parameters

\begin{tabular}{|c|c|}
\hline Parameter name & Value \\
\hline Number of samples $(M)$ & $250,500,1000$ \\
\hline SNR & $-20 \mathrm{~dB}-0 \mathrm{~dB}$ \\
\hline Sensing Interval & $50 \mu \mathrm{s}$ \\
\hline Sigma $(\sigma)$ & 1 \\
\hline Mobility & freeway mobility model \\
\hline
\end{tabular}

The detection probability is compared with the threshold for the number of samples in consideration. Fig. 3 helps in determining the optimal SNR given different sensing sample size.

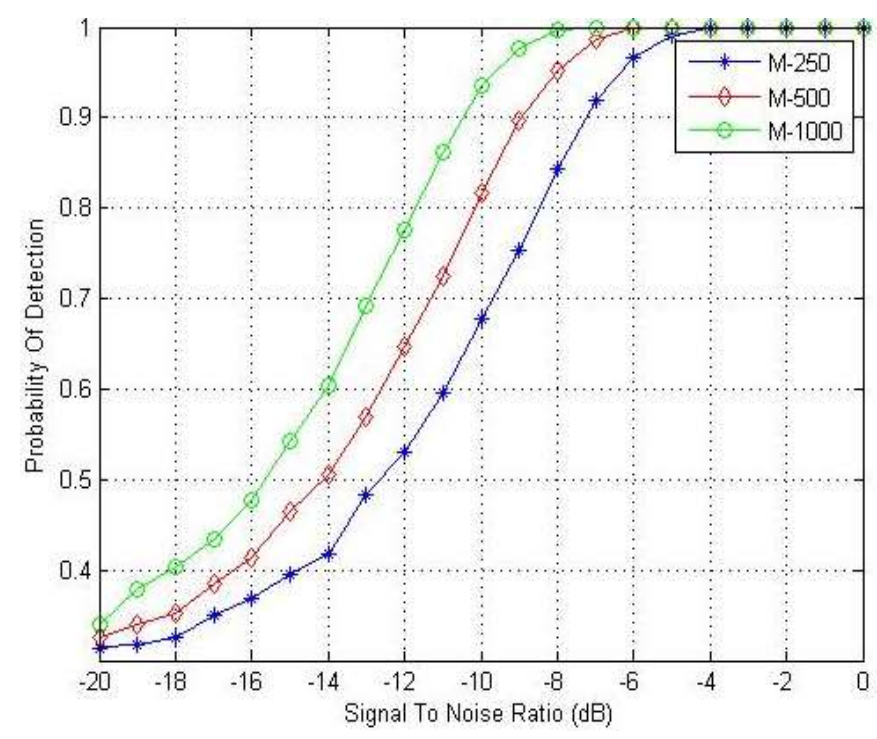

Fig. 3: Probability of detection verse SNR

The goal of Fig. 3 is to determine the optimal SNR that yields the maximum detection probability for the energy detector. As shown in Fig. 3, the energy detector performs poorly in low SNR. For instance, when the SNR is less that $-16 \mathrm{~dB}$, it is hard for the SU to determine whether the PU is present or absent because the probability of detection is very low. On the other hand, an increment in the number of samples to sense over time increases the detection probability. Cooperative decision can be used in low SNR to accurately identify the PU signal. Vehicles use spatial and diversity gain to overcome shadowing and multipath fading.

\subsection{Performance of SVM Kernel Functions}

The performance of SVM is evaluated using MATLAB. Before online classification, the RSU goes through training phase to correctly categorize subsequent test energy levels into the correct group (i.e., for the presence of PU ( $Y=$ 1) and PU absence $(Y=-1)$ as in Eq. 6). The training is done offline, hence it can be performed by RSU at regular intervals without affecting the performance of decision making. Different training samples were obtained from the vehicles' energy test level used in training the RSU. The table below presents the time to train and classify each kernel with training samples $(N=100,200,500)$ as well as the accuracy of classification. 
Table 2: SVM Kernel training, classification time in seconds and classification accuracy

\begin{tabular}{|l|c|c|c|c|}
\hline & $\begin{array}{c}\text { TRAINING } \\
\text { SAMPLE SIZE }\end{array}$ & $\begin{array}{c}\text { LINEAR- } \\
\text { SVM }\end{array}$ & $\begin{array}{c}\text { POLYNOMIAL- } \\
\text { SVM }\end{array}$ & RBF-SVM \\
\hline \multirow{3}{*}{$\begin{array}{l}\text { Kernel Training } \\
\text { Time (Seconds) }\end{array}$} & 100 & 0.215 & 0.204 & 0.091 \\
\cline { 2 - 5 } & 200 & 0.632 & 0.204 & 0.201 \\
\hline $\begin{array}{l}\text { Kernel } \\
\text { Classification } \\
\text { Time (Seconds) }\end{array}$ & 500 & 10.072 & 9.901 & 1.683 \\
\cline { 2 - 5 } $\begin{array}{l}\text { Classification } \\
\text { Accuracy (\%) }\end{array}$ & 100 & $1.21 \mathrm{E}-6$ & $1.37 \mathrm{E}-6$ & $7.29 \mathrm{E}-6$ \\
\cline { 2 - 5 } & 200 & $2.37 \mathrm{E}-6$ & $1.10 \mathrm{E}-6$ & $5.11 \mathrm{E}-6$ \\
\hline
\end{tabular}

The RBF kernel follows a Gaussian distribution and performs better than linear and polynomial kernels. This is because the spectrum sensing in energy detection follows the Gaussian distribution and the samples are not linearly separated as suggested in Section 3. In Fig. 4, the performance of each kernel is evaluated on the ROC curves.

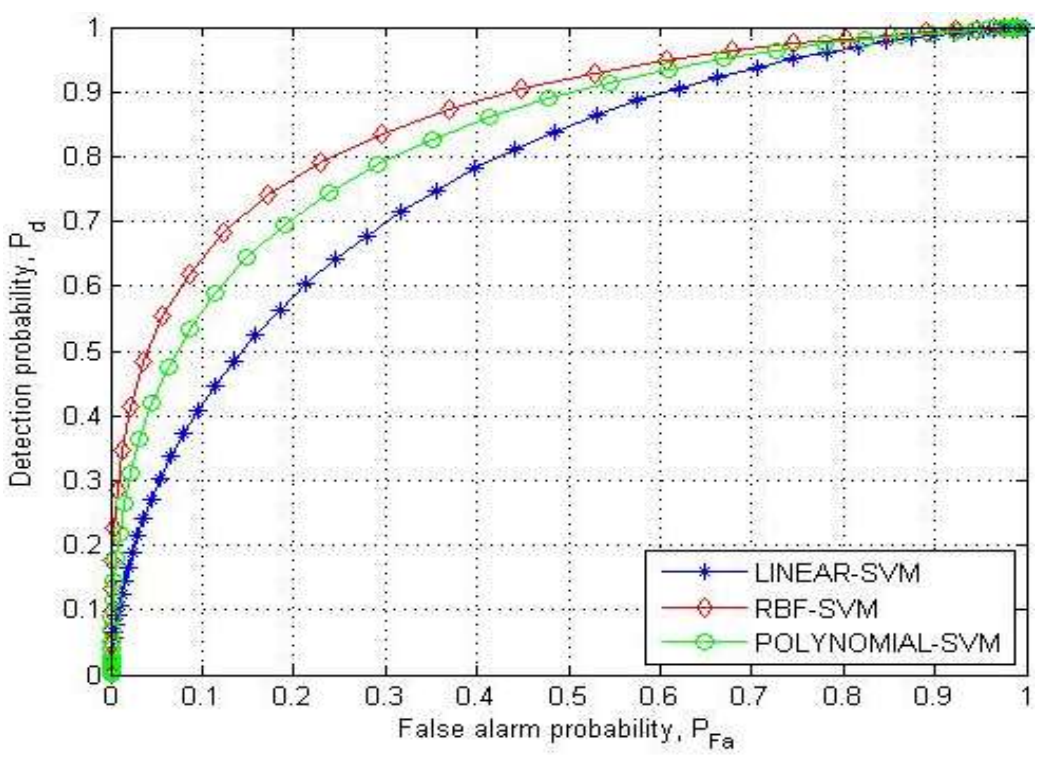

Fig. 4: Kernel function detection performance under ROC

In Fig. 4, RBF outperforms other kernels in terms of probability of detection. However, the classification time for $\mathrm{RBF}$ is poor as compared to the other kernels as noted in Table II. For instance, it takes 6.87E-6 seconds to classify 500 training samples with linear kernel, on the other hand, it takes 3.93E-5 seconds to classify the same samples. However, the classification time is negligible compared to the sensing interval of $50 \mu \mathrm{s}$. Hence, the effect on spectrum sensing and transmission time is minimal (see Section 3.2). In Fig. 5, the simulation was conducted to evaluate the performance of each kernel with an increasing number of vehicles participating in the sensing. The SNR is set to $-8 \mathrm{~dB}$. 


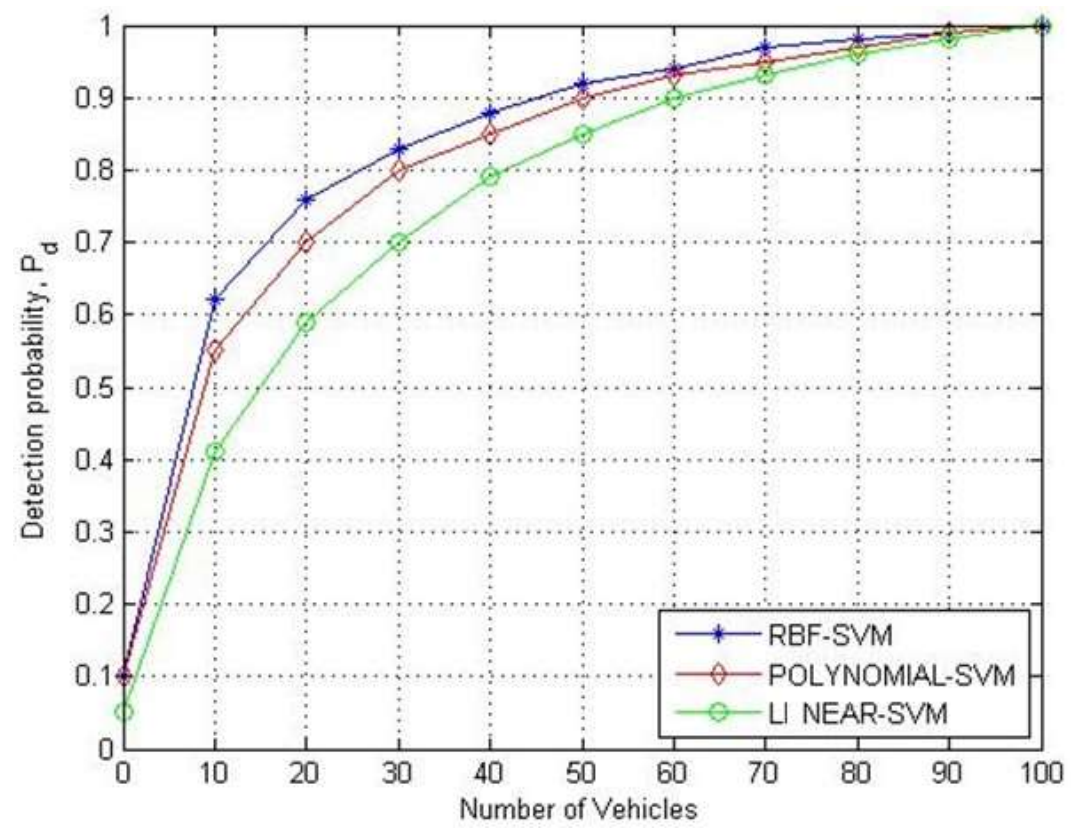

Fig. 5: Detection probability with different number of vehicles

The RBF kernel performs better than linear and polynomial kernels as seen from the figure above. With 10 vehicles participating in the cooperative decision, the detection probability is about $62 \%$ compared to $55 \%$ and $41 \%$ for polynomial and linear kernels respectively. Nevertheless, with an increasing number of vehicles participating in sensing, all kernel functions performance improves. In Fig. 6 and 7, we compare the performance of SVM based model to hard fusion rule from literature [33] [17] [34]. In the hard combining scheme, each vehicle senses the channel of interest and sends the binary output to the RSU. The binary outputs are 1 (indicating presence of the PU) and 0 (denoting absence of the PU). The RSU decides on spectrum occupancy using K-out-of-M rule where decide 1 if $\mathrm{K}$ out of $\mathrm{M}$ vehicles sent 1 as their output.

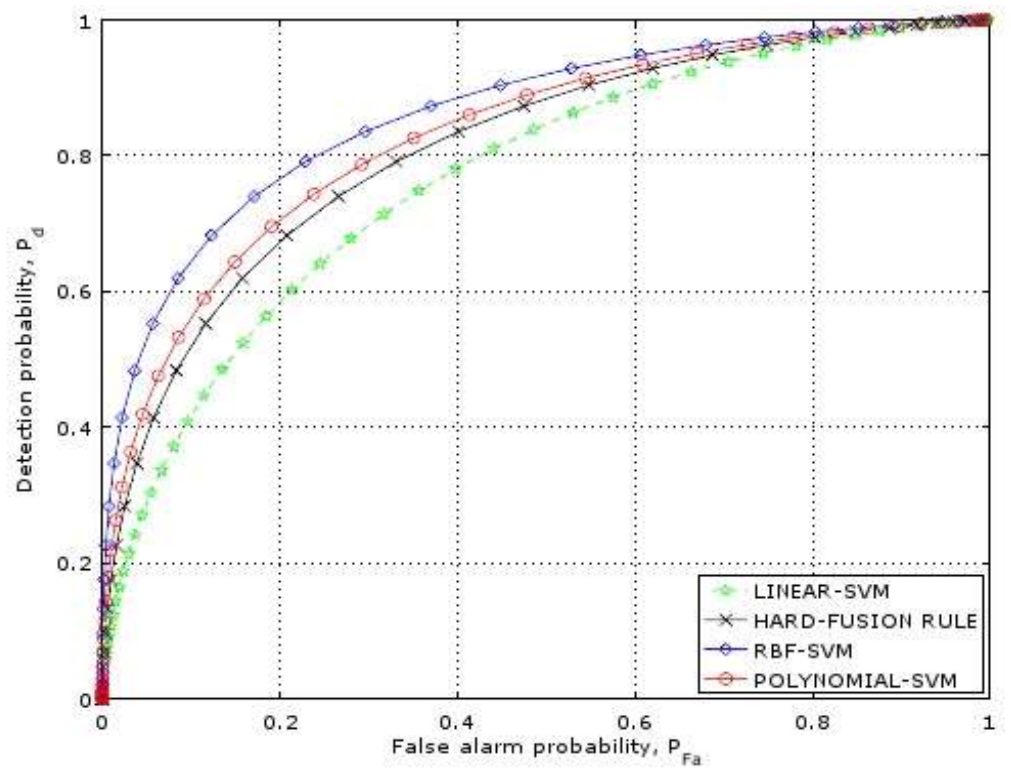

Fig. 6: ROC for SVM and hard fusion rule

The simulation results presented in Fig. 6 for ROC shows that RBF and polynomial kernel based SVM cooperative decision methods perform better than hard fusion and linear SVM. Hard fusion requires the participating vehicles to send only a bit to the RSU. On the other hand, SVM requires the vehicle to send the energy statistic level and the label for correct categorization. In addition, training the SVM increases its performance which is lacked in hard 
fusion rule. Nevertheless, hard fusion rule performs better than SVM model based on the linear kernel. More simulation results which compare the performance of SVM and hard fusion rule are presented in Fig. 7.

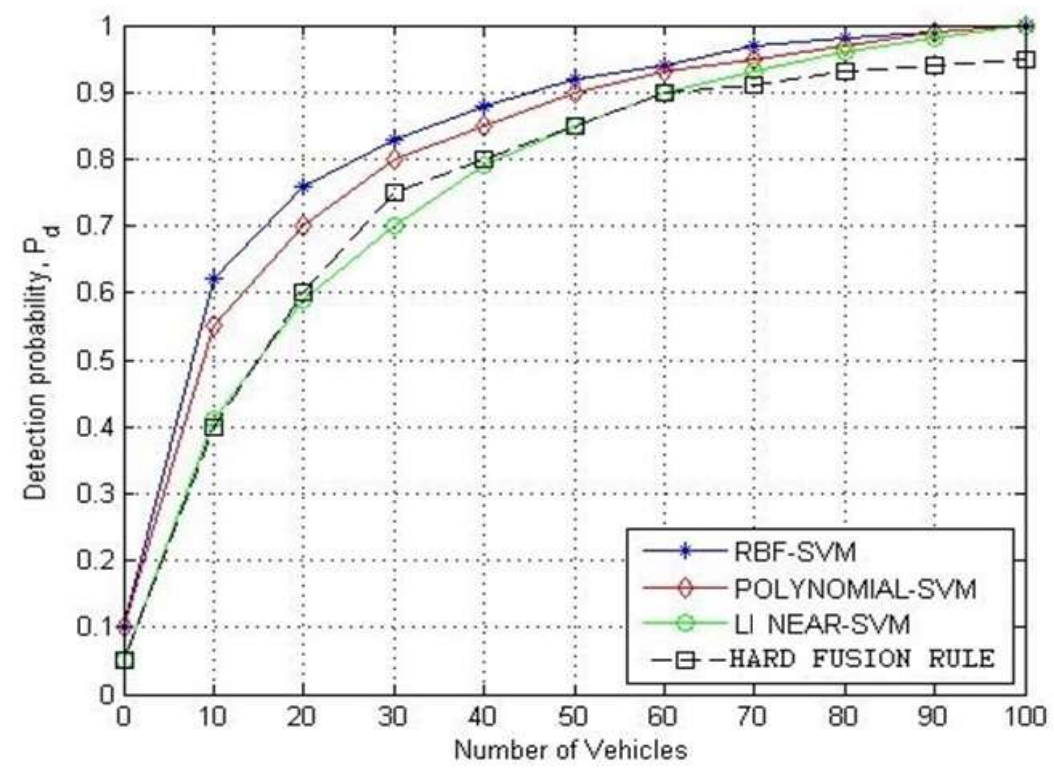

Fig. 7: Comparison of SVM and hard fusion rule

As shown in Fig. 7, the hard fusion rule scheme performs below the RBF and Polynomial SVM based techniques. For instance, it takes 45 vehicles when using RBF to reach the detection probability of $90 \%$ while the hard fusion requires 60 vehicles for the same SNR $=-10 \mathrm{~dB}$. Furthermore, all the SVM kernels perform better with the increased number of vehicles to reach a desired detection probability compared to hard fusion rule method. For example, in Fig. 7, all the kernel based sensing reach at least $99 \%$ detection probability for 90 vehicles when using $\mathrm{SNR}=-10$ while the hard fusion detection probability is at $95 \%$. The implication is that the SVM based decision at RSU performed better than hard fusion rule approach. One of the shortcomings of SVM based approach is that it requires training before sensing can be performed online. In addition, online detection is associated with the particular channels which the model is trained on. Nevertheless, as more data is collected on many channels, the sensing performance for many channels can be achieved and improved upon to cover more channels in the learning process.

\subsection{CONCLUSION}

In this paper, we have shown that cooperative spectrum decision based on support vector machine performs better than hard fusion rule decision at RSU. Simulation results indicate that RBF outperforms linear and polynomial kernels. In general, however, the proposed SVM based sensing performs better than hard fusion combining rule approach in low SNR in terms of detection probability. For example, RBF SVM based approach reach the probability of detection of $75 \%$ while hard fusion rule is about $60 \%$ with 20 vehicles participating in the cooperative decision. With an increase in the number of vehicles the probability of decision also increases but at different rates. In dynamic spectrum access, spectrum sensing is one of the most important stages. Accurate identification of free licensed channel will reduce interference to primary systems. In addition, accurate sensing results will enable optimal use of free licensed channels whenever they are free. Therefore, the results obtained in this paper show that support vector machine based decision making can play an important role in identifying free spectrum from licensed frequency bands along the roads. In the future, we will investigate other machine learning techniques and their influence on detecting the PU signal. Furthermore, we will also study other PU activity model and their effect on sensing performance in $\mathrm{CVN}$ environment.

\section{ACKNOWLEDGMENT}

This research is supported by the Faculty Program Grant, GPF009D-2018. 


\section{REFERENCES}

[1] M. Oche, R. M. Noor, and J. I. Aghinya, "Network centric QoS performance evaluation of IPTV transmission quality over VANETs," Computer Communications, Vol. 61, May 2015, pp. 34-47.

[2] R. Kumar and M. Dave, "A review of various vanet data dissemination protocols," International Journal of $u$-and e-Service, Science and Technology, Vol. 5, No. 3, 2012, pp. 27-44.

[3] H. Sundmaeker, P. Guillemin, P. Friess, and S. Woelfflé, Vision and challenges for realising the Internet of Things Vol. 20: EUR-OP, 2010.

[4] J. R. Williams. U.S. Spectrum Allocations $300 \quad$ - $3000 \quad \mathrm{MHz}$. Available: http:/transition.fcc.gov/Bureaus/OPP/working_papers/oppwp38chart.pdf, 2002

[5] J. B. Kenney, "Dedicated short-range communications (DSRC) standards in the United States," Proceedings of the IEEE, Vol. 99, 2011, pp. 1162-1182.

[6] F. Qu, F.-Y. Wang, and L. Yang, "Intelligent transportation spaces: vehicles, traffic, communications, and beyond," Communications Magazine, IEEE, Vol. 48, No. 11, 2010, pp. 136-142.

[7] G. Samara and T. Alhmiedat, "Intelligent emergency message broadcasting in VANET using PSO," arXiv preprint arXiv:1406.7399, 2014.

[8] Z. Wang and M. Hassan, "How much of dsrc is available for non-safety use?," in Proceedings of the fifth ACM international workshop on VehiculAr Inter-NETworking, 2008, pp. 23-29.

[9] J. Mitola III and G. Q. Maguire Jr, "Cognitive radio: making software radios more personal," Personal Communications, IEEE, Vol. 6, No. 4, 1999, pp. 13-18.

[10] D. Das and S. Das, "A Survey on Spectrum Occupancy Measurement for Cognitive Radio," Wireless Personal Communications, Vol. 85, No. 4, December 2015, pp. 2581-2598.

[11] V. Sharma and V. A. Bohara, "Exploiting machine learning algorithms for cognitive radio," in Advances in Computing, Communications and Informatics (ICACCI, 2014 International Conference on, 2014, pp. 15541558.

[12] K. M. Thilina, K. W. Choi, N. Saquib, and E. Hossain, "Pattern classification techniques for cooperative spectrum sensing in cognitive radio networks: SVM and W-KNN approaches," in Global Communications Conference (GLOBECOM), 2012 IEEE, 2012, pp. 1260-1265.

[13] H. Li and D. K. Irick, "Collaborative spectrum sensing in cognitive radio vehicular ad hoc networks: belief propagation on highway," in 2010 IEEE 71st Vehicular Technology Conference, 2010.

[14] M. D. Felice, K. R. Chowdhury, and L. Bononi, "Cooperative spectrum management in cognitive vehicular ad hoc networks," in Vehicular Networking Conference (VNC), 2011 IEEE, 2011, pp. 47-54.

[15] W. Ejaz, N. Ul Hasan, and H. S. Kim, "Distributed cooperative spectrum sensing in cognitive radio for ad hoc networks," Computer Communications, Vol. 36, No. 12, 2013, pp. 1341-1349.

[16] K. Baraka, L. Safatly, H. Artail, A. Ghandour, and A. El-Hajj, "An infrastructure-aided cooperative spectrum sensing scheme for vehicular ad hoc networks," Ad Hoc Networks, Vol. 25, 2015, pp. 197-212.

[17] J.-Q. Duan, S. Li, and G. Ning, "Compressive Spectrum Sensing in Centralized Vehicular Cognitive Radio Networks," International Journal of Future Generation Communication and Networking, Vol. 6, 2013, pp. $1-12$.

[18] Y. Liu, S. Xie, R. Yu, Y. Zhang, X. Zhang, and C. Yuen, "Exploiting temporal and spatial diversities for spectrum sensing and access in cognitive vehicular networks," Wireless Communications and Mobile Computing, Vol. 15, 2015, pp. 2079-2094. 
[19] K. M. Thilina, K. W. Choi, N. Saquib, and E. Hossain, "Machine learning techniques for cooperative spectrum sensing in cognitive radio networks," Selected Areas in Communications, IEEE Journal on, vol. 31, 2013, pp. 2209-2221.

[20] Z. Dandan and Z. Xuping, "SVM-based Spectrum Sensing in Cognitive radio," in Wireless Communications, Networking and Mobile Computing (WiCOM), 2011 7th International Conference on, 2011, pp. 1-4.

[21] H. Yang, X. Xie, and R. Wang, "SOM-GA-SVM Detection Based Spectrum Sensing in Cognitive Radio," in Wireless Communications, Networking and Mobile Computing (WiCOM), 2012 8th International Conference on, 2012, pp. 1-7.

[22] O. P. Awe, Z. Zhu, and S. Lambotharan, "Eigenvalue and support vector machine techniques for spectrum sensing in cognitive radio networks," in Technologies and Applications of Artificial Intelligence (TAAI), 2013 Conference on, 2013, pp. 223-227.

[23] B. F. Lo and I. F. Akyildiz, "Reinforcement learning-based cooperative sensing in cognitive radio ad hoc networks," in Personal Indoor and Mobile Radio Communications (PIMRC), 2010 IEEE 21st International Symposium on, 2010, pp. 2244-2249.

[24] X. Qian and L. Hao, "Spectrum sensing with energy detection in cognitive Vehicular Ad hoc Networks," in Wireless Vehicular Communications (WiVeC), 2014 IEEE 6th International Symposium on, 2014, pp. 1-5.

[25] V. Tyagi, S. Kalyanaraman, and R. Krishnapuram, "Vehicular traffic density state estimation based on cumulative road acoustics," Intelligent Transportation Systems, IEEE Transactions on, Vol. 13, 2012, pp. 1156-1166.

[26] J. Lee and C. M. Kim, "A roadside unit placement scheme for vehicular telematics networks," in Advances in Computer science and information technology, ed: Springer, 2010, pp. 196-202.

[27] Y. Zeng, Y.-C. Liang, A. T. Hoang, and R. Zhang, "A review on spectrum sensing for cognitive radio: challenges and solutions," EURASIP Journal on Advances in Signal Processing, Vol. 2010, pp. 1-15, 2010.

[28] D. Cabric, A. Tkachenko, and R. W. Brodersen, "Experimental study of spectrum sensing based on energy detection and network cooperation," in Proceedings of the first international workshop on Technology and policy for accessing spectrum, 2006, p. 12.

[29] C. Cortes and V. Vapnik, "Support-vector networks," Machine learning, Vol. 20, 1995, pp. 273-297.

[30] N. Swetha, P. N. Sastry, and Y. R. Rao, "Analysis of Spectrum Sensing Based on Energy Detection Method in Cognitive Radio Networks," in IT Convergence and Security (ICITCS), 2014 International Conference on, 2014, pp. 1-4.

[31] F. F. Digham, M.-S. Alouini, and M. K. Simon, "On the energy detection of unknown signals over fading channels," IEEE transactions on communications, Vol. 55, 2015, pp. 21-24.

[32] S. Atapattu, C. Tellambura, and H. Jiang, "Energy detection based cooperative spectrum sensing in cognitive radio networks," Wireless Communications, IEEE Transactions on, Vol. 10, 2011, pp. 12321241.

[33] X. Xu, A. Huang, J. Zhang, and L. Gu, "Cooperative wideband spectrum detection based on Maximum Likelihood Ratio for cognitive VANET," in Automatic Control and Artificial Intelligence (ACAI 2012), International Conference on, 2012, pp. 726-729.

[34] D. Borota, G. Ivkovic, R. Vuyyuru, O. Altintas, I. Seskar, and P. Spasojevic, "On the delay to reliably detect channel availability in cooperative vehicular environments," in Vehicular Technology Conference (VTC Spring), 2011 IEEE 73rd, 2011, pp. 1-5. 
[35] X.-L. Huang, J. Wu, W. Li, Z. Zhang, F. Zhu, and M. Wu, "Historical Spectrum Sensing Data Mining for Cognitive Radio Enabled Vehicular Ad-Hoc Networks," IEEE Transactions on Dependable and Secure Computing, Vol. 13, 2016, pp. 59-70.

[36] F. P. Release, Second Memorandum Opinion and Order (FCC 10- 174), ET Docket Nos. 02-380 and 04186, September 2010.

[37] M. Di Felice, A. J. Ghandhour, H. Artail, and L. Bononi, "Integrating spectrum database and cooperative sensing for cognitive vehicular networks," in Vehicular Technology Conference (VTC Fall), 2013 IEEE 78th, 2013, pp. 1-7.

[38] C. Chembe, R.M. Noor, and E. Mostajeran, "Optimizing wireless channel using adaptive modulation to improve QoS in VANET," Malaysian Journal of Computer Science, Vol. 26 No. 1, 2013, pp.34-43.

[39] C. Chembe, RM Noor, I. Ahmedy, M. Oche, D. Kunda, C. H. Liu, "Spectrum sensing in cognitive vehicular network: State-of-Art, challenges and open issues". Computer Communications. Vol 97, No. 1, January 2017, pp. 15-30. 